\title{
Effects of continuum of care in maternal health services on adverse birth outcome in Northwest Ethiopia: a prospective follow up study
}

Muluwas Amentie Zelka ( $\square$ muluwas12@gmail.com )

Addis Ababa University School of Public Health https://orcid.org/0000-0003-4298-7061

Alemayehu Worku Yalew

Addis Ababa University School of Public Health

Gurmesa Tura Debelew

Jimma Institute of Health Sciences: Jimma University

\section{Research Article}

Keywords: stillbirth, neonatal death, perinatal death, maternal health, continuum of care

Posted Date: November 11th, 2021

DOl: https://doi.org/10.21203/rs.3.rs-1069811/v1

License: (c) (i) This work is licensed under a Creative Commons Attribution 4.0 International License.

Read Full License 


\section{Abstract \\ Background}

Globally, around 4 million of babies were died within the first month and more than 3 million were stillbirth. Of them, $99 \%$ of newborn deaths and $98 \%$ of stillbirth occur in developing countries. Despite give a priority for maternal health services, adverse birth outcomes are still major public health problems and big challenge in Ethiopia particularly in study area. Hence, to overcome those challenges, a continuum of care (COC) is core key strategies. Despite that study conduct on effectiveness of $\mathrm{COC}$ in maternal health services was scare in developing countries and not done in study area. We aimed to assess effectiveness of continuity of care on reduction of adverse events and determinants of adverse birth outcomes.

\section{Methods}

Community and health facility linked prospective follow-up study design was employed from March 2020 to January 2021 in Northwest Ethiopia. A multistage sampling technique was used to recruit 2,198 pregnant women. Data were collected by using semi-structured and pretested interviewer questionnaire. Collected data were coded, entered, cleaned and analyzed by using STATA 14. Mixed-effect model was used to identify community and individual level factors of adverse birth outcomes. Finally, propensity score matching (PSM) was applied to determine effectiveness of COC in maternal health services.

\section{Results}

Magnitude of adverse birth outcome was $26.8 \%$ : stillbirth (2.7\%), neonatal mortality rate (3.1\%) and neonatal morbidity (6.8\%). Risk factors significantly associated were being poor household wealth index $(A O R=3.3)$, pregnant related complication during pregnancy $(A O R=3.29)$, labour and childbirth $(A O R=$ 6.08), immediately after childbirth $(A O R=5.24)$, offensive odder amniotic fluid $(A O R=3.04)$ and history of stillbirth $(A O R=4.2)$. Whereas, women receive IFA during pregnancy $(A O R=0.44)$, initiate $B F$ within 1 hour $(A O R=0.22)$ and immunizing newborn within postnatal period $(A O R=0.33)$ were protective factors. As treatment effect, completion of $\mathrm{COC}$ in MHS via time dimension $(\beta=-0.03)$, completion of key services of MHS $(\beta=-0.04)$ and completion of $\mathrm{COC}$ in MHS via space dimension $(\beta=-0.02)$ were associated with a significant reduction in the likelihood of perinatal death

\section{Conclusions}

The results suggested that neonatal and perinatal mortality rate were high as compared with national and international target. Completion of $\mathrm{COC}$ in MHS is an effective strategy in reduction perinatal death. 
Efforts should be made to strengthening COC of MHS and also encourage IFA supplementation, immunizing and early initiation of BF for newborn were strongly recommended.

\section{Background}

Women play a significant role in rearing of children and managing family affairs but their loss from maternity-related causes and neonatal mortality are a significant social, economic and personal disaster(1). Globally, around 4 million of babies were died within the first month and more than 3 million of births were stillbirth. Hence, $99 \%$ of newborn deaths occur in developing countries(2). It is a big challenge for the world population and still public health problem particularly in the developing and lowincome countries(3). In Africa, annually 1 million stillbirths were occurred, of them 300,000 cases were occurred during labour and also 1 million babies were died within first month, of them half million died within 1 st day. In Sub-Sahara Africa, under five child mortality rate was 16 times more likely to die than in high income countries due to poor access to quality of services and discontinuation of care in maternal health services $(2,4)$. Having this in mind, those deaths were peak during the times of birth, the first day and the first week of life.

The health need of mother and newborn are inseparably linked together. In the past decay: maternal, newborn and child health policy and programs address their health need separately; this gap and challenge in care provision especially newborn health are subjected to disadvantaged. Even though, to overcome those challenges, a continuum of care ( $\mathrm{CoC})$ in maternal health services is one of the core key strategies. Thus, it reaches mothers and babies at a crucial time and place to reduce the risk of death, illness or disability of neonatal (5). Health of newborn relies on good linkage of maternal health intervention because newborn health is a sensitive indicator of continuity of maternal health services (5). Despite these, almost half of African women were not receiving skilled care during childbirth and postnatal care services after delivery(4).

While utilization rate of essential maternal health services reach $90 \%$, neonatal death reduced by $37-$ $67 \%(430,000-800,000)$ of deaths $(4)$. Hence, continuum of care in maternal health services via time dimension could reduce neonatal mortality: completion of continuity of Antenatal (ANC) services could reduce by $7-14 \%$; continuity of care at child birth could reduce by $19-34 \%$ and continuity of postnatal (PNC) services could reduce $10-27 \%$ of neonatal deaths. Similarly, space dimension continuity of care could avert neonatal death: continuity of care at family/community level interventions $(14-32 \%)$; outpatient/outreach interventions $(7-14 \%)$ and clinical setting interventions ( $26-51 \%)$ of neonatal death could avert(5). A variety of evidence revealed that completion of continuity of care in maternal health services via time and space dimension were more likely reduce adverse birth outcomes (stillbirth, neonatal mortality and morbidity) $(4,6-12)$.

Despite give a priority for maternal health services, adverse birth outcome (stillbirth, neonatal death and illness) are still major public health problems in Ethiopia particularly in Benishangul Gumuz Region. However, majority of literature suggested evidences based on cross sectional study design and facility 
based survey which couldn't show a cause and effect relationship and also using traditional binary logistic and linear regression couldn't explore the treatment effect of completion of continuity of maternal health services on adverse birth outcome. Moreover, relies on cross sectional and facility survey, it is difficult to organize clear epidemiological evidence on treatment effect. In general, clear epidemiological data on adverse birth outcome (stillbirth, neonatal death and morbidity) were generated using prospective follow up study design, which found to be an important evidence for implementation of maternal health services in Ethiopia.

Therefore, the objective of this study was to investigate effect of continuum of care in maternal health services on perinatal death and also determinant factors that hindering adverse birth outcome using propensity score matching (PSM) and multilevel regression model respectively.

\section{Methods}

\section{Study design and setting}

Community and health facility linked prospective follow-up study design was conducted in BenishangulGumuz Regional State (BGRS) from March 2020 to January 2021. The region is one of the eleven states of Ethiopia's Federal Democratic Republic of Ethiopia. Assosa town is the capital city of the region, located $670 \mathrm{kms}$ West of Addis Ababa, the capital city of Ethiopia. The region has three zones, three town administrative cities, twenty one districts/ Woredas, one special district/ Woreda's and 475 clusters/ Kebele's (439 rural and 36 urban clusters/ Kebele's). The region represents around $4.6 \%$ of the total land area of Ethiopia and most of the people in the region are sparsely populated (13).

\section{Source population and study participants}

All pregnant women and birth that registered as live birth as well as stillbirth at the time of birth within the follow up period were considered as source population. Whereas, the study population was newborns that registered as "live birth" or "stillbirth" (which is declared by women, birth attendants or health workers) at the time of birth and selected by simple sampling techniques.

\section{Sample size and sampling technique}

The sample size for this study was calculated by using STATA/MP 13.0 software by considering two population proportion formulas based on the following assumption. The outcome variable adverse birth outcome (stillbirth, neonatal death and any illness within neonatal period), and the predictor variable was continuum of care in maternal health service. In Ethiopia, literatures that support to determine sample size for this objective are not available. Like as Ethiopia particularly in Benishangul Gumuz Region; in rural India, most births take place in the home, where high-risk practices are common(10). Unfortunately, neonatal mortality in Uttar Pradesh, India (29 deaths per 1,000live birth: Urban 20 verses Rural 38 deaths 
per 1,000live birth)(14) is almost similar with Ethiopia neonatal mortality (29 per 1,000live birth) (15), so that evidence stated in Uttar Pradesh, India is used for sample size determination. Study done in Uttar Pradesh, India was found that proportion of adverse birth outcome, "neonatal death", among whose mother use complete continuum of care in maternal health services is $4.29 \%\left(\left(P_{1}=0.0429\right)\right.$ and proportion of adverse birth outcome, "neonatal death", among whose mother never use ANC service is $8.43 \%\left(P_{2}=0.0843\right)(10)$. A $95 \%$ confidence level and $80 \%$ power were used to detect $9.8 \%$ difference or $54.4 \%$ increment among exposed and non-exposed group. Hence, $\mathbf{r}=$ ratio of exposure to non-exposure pregnant women equal to 1:1 for the population allocation ratio; $\mathbf{P}$ (pooled population proportion) $=\frac{P_{1}+P_{2}}{1+r}$

was calculated ( $P=0.0636)$; considering a design effect 2 and a non-response rate of $10 \%$. As result, the final sample size was 2,402 pregnant women had been follow up to look the effect of continuity of maternal health services on perinatal death, used for as final sample size for this study.

Since this research work was carried out at a regional level, the study subjects (pregnant women) were chosen using a multistage sampling technique. The sampling procedure used for this study was as follow: primarily two zones and one town administration were chosen by simple random sampling (SRS). Following that, four districts/“woredas" from the Assosa Zone, two districts/"woredas" from the Metekel Zone, and two districts/"woredas" from the Assosa town administration were chosen by simple random sampling (SRS) technique. Thirdly, from each selected district/“ woreda", seven kebeles from each district (except Assosa district/" woreda": 10 kebeles were selected) and five ketenes from each district/“woreda" of town administration were selected and included in the study. Then after, among the selected kebeles/ketenas (7 kebeles from each districts/"woredas", 10 kebeles from Assosa districts/"woredas" and five ketenes from each districts/"woredas" of town administration), pregnant women were enumerated by using house-to house visit and all obtained and registered pregnant women were included in the study. All women who claimed an 8 weeks or longer pregnancy, as determined by the loss of two consecutive menses, were considered eligible and joined in the study, which followed for 11 months. Assuming that each household with pregnant women had at least one pregnant woman, households with pregnant women and neonates were selected as the final sampling unit (FSU). Similarly, all health facilities found within the catchment areas were list and considered as a candidate for health facility survey. Therefore, 46 health facilities ( 3 hospitals, 12 health centers and 31 health posts) were found within the catchment areas and included for health facility survey.

\section{Data collection process}

Data collection was conducted using semi-structured questionnaires and registration format adopted from EDHS 2016(15), National Technical Guidance for MPDSR 2017(16), MCH Program Indictor Survey 2013(17), Survey tools conduct in Jimma Zone, Southwest Ethiopia(18), Survey tools conducted in Rural South Ethiopia(19) and other relevant different literatures. The instrument was prepared in English and translated into local language ("Amharic") and then back translated to English to ensure validity of the 
instrument. Following that, training was offered for data collectors and supervisors for 3 days and also pre-test was carried out on 35 individuals, located outside of the study areas/cluster. During actual data collection, principal investigator and supervisors were frequently supervised and checked the work of data collectors and also clarification and direction was forwarded to those who had doubts.

\section{Data management and analysis}

Data was coded and entered into Epi Info version 7.2.2.6 to develop skipping patterns and avoid logical mistakes. The data was then cleaned, edited, and analyzed using STATA Software version 14. All variables were computed for descriptive statistics. Analysis with only one variable were performed, the crude odds ratio and $95 \%$ confidence interval were used to select candidate variables for multivariate analysis $(p<0.25)$. At the level of significance $(p<0.05)$, a maximum likelihood estimate of the independent effect on the outcome variable was calculated. The household wealth index was calculated and categorized by using Principal Component Analysis (PCA). Before running the full model, effect modification or interaction effect at $p<0.1$ and multi-collinarity effect between independent variables using variance inflation factors (VIF > 10\%) were assessed. All independent variables included had VIP $<$ 10 and multi-collinearity effect of each variables were $p<0.1$. Hence, there was no significant interaction and multi-collinearity effect was detected. Since, the sampling procedure for this study was multistage sampling procedure; due to cluster variability mixed effect multilevel regression model was applied to detect determinants factors of adverse birth outcomes (still birth, neonatal death and any neonatal illness). Thus, for this study 'Kebeles/Ketenas' were categorized as clusters and cluster level variables were place of residence, access to health center and household wealth index were taken as level -2 factors. Women who gave birth during follow study were nested within their households and therefor in the community. As a result women individual level variables were socio-demographic, obstetric, information, maternal health services and newborn health services were taken as level - 1 factor. Log likelihood ratio (LR) test was to confirm goodness of fit of the multilevel model that found to be statistical significant such as data fit the model. Finally, the effect of continuity of maternal health services perinatal death was estimated by propensity score matching. The effect was measured by $\beta 95 \% \mathrm{Cl}$ at $p<$ 0.05 .

\section{Results}

\section{Newborn Characteristics and Health Related Issues}

The characteristics of newborns born: 1102 (53.7\%) were male and 359 (17.4\%) were amniotic member break before labour started and time of premature rupture of membrane was 1 - 12 hours before labour started 1132 (55.5\%). More than three fourth 1585 (76.8\%) of women reported that the color of amniotic fluid was clear, whereas 305 (14.8\%) of women stated that amniotic fluid had bad odor. Majority, 1896 $(91.8 \%)$ was the baby ever breast fed within 28 days and 1851 (84.2\%) of the newborns were crying immediately after birth (Table 1). 
Table 1: Newborn health related characteristic in Benishangul Gumuz Region, Northwestern Ethiopia, March 2020 - January 2021

\begin{tabular}{|c|c|c|}
\hline Variables & Frequency & Percent (\%) \\
\hline $\begin{array}{c}\text { Sex of newborn }(n=2053) \\
\text { Male } \\
\text { Female }\end{array}$ & $\begin{array}{l}1102 \\
951\end{array}$ & $\begin{array}{l}53.7 \\
46.3\end{array}$ \\
\hline $\begin{array}{c}\text { Time of amniotic member break } \\
\text { Before labour started } \\
\text { During labour } \\
\text { Unknown }\end{array}$ & $\begin{array}{l}359 \\
1674 \\
32\end{array}$ & $\begin{array}{l}17.4 \\
81.1 \\
1.5\end{array}$ \\
\hline $\begin{array}{c}\text { Time of premature rupture of membrane } \\
\qquad=1 \text { hour before labour } \\
1-12 \text { hours before labour } \\
>12 \text { hours before labour }\end{array}$ & $\begin{array}{l}814 \\
1132 \\
94\end{array}$ & $\begin{array}{l}39.9 \\
55.5 \\
4.6\end{array}$ \\
\hline $\begin{array}{l}\text { Color of amniotic fluid } \\
\text { Clear } \\
\text { Yellow } \\
\text { Green } \\
\text { Brown } \\
\text { Dark red } \\
\text { Unknown } \\
\end{array}$ & $\begin{array}{l}1585 \\
150 \\
33 \\
88 \\
107 \\
102 \\
\end{array}$ & $\begin{array}{l}76.8 \\
7.3 \\
1.6 \\
4.3 \\
5.2 \\
4.9 \\
\end{array}$ \\
\hline $\begin{array}{c}\text { Did the water/amniotic fluid smell bad } \\
\text { Yes } \\
\text { No }\end{array}$ & $\begin{array}{l}306 \\
1758 \\
\end{array}$ & $\begin{array}{l}14.8 \\
85.2 \\
\end{array}$ \\
\hline $\begin{array}{c}\text { Was the baby ever breast feed within 28days? } \\
\text { Yes } \\
\text { No }\end{array}$ & $\begin{array}{l}1896 \\
169\end{array}$ & $\begin{array}{l}91.8 \\
8.2\end{array}$ \\
\hline $\begin{array}{c}\text { Did the baby cry immediately after birth } \\
\text { Yes } \\
\text { No }\end{array}$ & $\begin{array}{l}1851 \\
214\end{array}$ & $\begin{array}{l}84.2 \\
9.7\end{array}$ \\
\hline
\end{tabular}

\section{Newborn Health Outcomes and Adverse Birth Outcomes}

Even though a total of 2065 pregnant women gave birth, 248 (12.4\%) were accompanied with adverse birth outcomes (stillbirth, 52 (2.7\%), neonatal death, 58 (3.1\%) and any neonatal illness with in postnatal period, 138 (6.8\%). Among newborns who died within postnatal periods, 35 (59.5\%) were died within 48 hours and $43(72.9 \%)$ of neonatal deaths were classified as early neonatal deaths. The three major neonatal characteristics that caused neonatal death: birth asphyxia 38 (66.0\%), sepsis/infection 26 $(50.5 \%)$ and pneumonia $22(42.0 \%)$. Similarly, the three major maternal characteristics cause neonatal death were obstructed labour 30 (56.4\%), obstetric sepsis/maternal infection 29 (54.7\%), and antepartum hemorrhage (APH) 16 (33.3\%).

After weight for cluster variation, among stillbirth, 24 (39.7\%) of stillbirth were takes place in hospital followed by health center 20 (37.4\%) and also around two third 38 (64.3\%) were attended by 
midwifery/nurse/health officers. The major possible cause of stillbirth were maternal infection/sepsis 38 (80.7\%), maternal malnutrition 15 (41.2\%) and unnecessary medication $12(17.8 \%)$. The top three pregnant related problems during pregnancy before the occurrence of stillbirth were severe abdominal pain 31 (66.2\%), excessive vaginal bleeding $23(50.1 \%)$ and blurred vision $11(31.6 \%)$. Whereas, common illness that women encountered during pregnancy were febrile illness $25(62.0 \%)$, malnutrition 7 (13.3\%) and anemia 5 (13.0\%) (Table 2).

Table 2: Adverse birth outcome and related factors among the study subject in Benishangul Gumuz Region, Northwestern Ethiopia, March 2020 - January 2021 


\begin{tabular}{|c|c|c|c|}
\hline Variables & Frequency & $\begin{array}{c}\text { Un-weighted } \\
\%\end{array}$ & $\begin{array}{c}\text { Weighted } \\
\%\end{array}$ \\
\hline $\begin{array}{l}\text { Adverse birth outcome } \\
\text { No } \\
\text { Yes }\end{array}$ & $\begin{array}{l}1817 \\
248\end{array}$ & $\begin{array}{l}87.99 \\
12.01\end{array}$ & $\begin{array}{l}87.6 \\
12.4\end{array}$ \\
\hline $\begin{array}{l}\text { Neonatal health outcome within 28days } \\
\text { Neonatal well being } \\
\text { Neonatal morbidity } \\
\text { Neonatal Mortality }\end{array}$ & $\begin{array}{l}1817 \\
138 \\
58\end{array}$ & $\begin{array}{l}90.3 \\
6.9 \\
2.9\end{array}$ & $\begin{array}{l}90.1 \\
6.8 \\
3.1\end{array}$ \\
\hline $\begin{array}{l}\text { Age of Neonatal death occurred }(\mathrm{n}=58) \\
\text { Within } 2 \text { days } \\
2-7 \text { days } \\
7 \text { - 28days } \\
\end{array}$ & $\begin{array}{l}35 \\
8 \\
15\end{array}$ & $\begin{array}{l}60.3 \\
13.8 \\
25.9\end{array}$ & $\begin{array}{l}59.5 \\
13.4 \\
27.1\end{array}$ \\
\hline $\begin{array}{l}\text { Classification of neonatal death } \\
\text { Early neonatal death } \\
\text { Late neonatal death }\end{array}$ & $\begin{array}{l}43 \\
15\end{array}$ & $\begin{array}{l}74.1 \\
25.9\end{array}$ & $\begin{array}{l}72.9 \\
27.1\end{array}$ \\
\hline $\begin{array}{l}\text { Neonatal characteristic cause neonatal death } \\
\text { Asphyxia } \\
\text { Sepsis } \\
\text { Pneumonia } \\
\text { Prematurity } \\
\text { Meningitis } \\
\text { Neonatal tetanus } \\
\text { Lethal congenital anomaly }\end{array}$ & $\begin{array}{l}38 \\
26 \\
22 \\
11 \\
1 \\
4 \\
18\end{array}$ & $\begin{array}{l}65.5 \\
44.8 \\
37.9 \\
19.0 \\
1.7 \\
6.9 \\
31.0\end{array}$ & $\begin{array}{l}66.0 \\
50.5 \\
42.0 \\
22.5 \\
2.4 \\
7.7 \\
33.0\end{array}$ \\
\hline $\begin{array}{l}\text { Maternal characteristic cause neonatal death } \\
\text { Obstructed labour } \\
\text { Obstetric sepsis } \\
\text { APH } \\
\text { Ruptured uterus } \\
\text { Preeclampsia/eclampsia } \\
\end{array}$ & $\begin{array}{l}30 \\
29 \\
16 \\
9 \\
8\end{array}$ & $\begin{array}{l}51.5 \\
50.0 \\
27.6 \\
15.5 \\
13.8\end{array}$ & $\begin{array}{l}56.4 \\
54.7 \\
33.3 \\
15.3 \\
17.4\end{array}$ \\
\hline $\begin{array}{l}\text { Birth encountered by stillbirth }(n=52) \\
\text { No } \\
\text { Yes }\end{array}$ & $\begin{array}{l}2146 \\
52\end{array}$ & $\begin{array}{l}97.6 \\
2.4\end{array}$ & $\begin{array}{l}97.3 \\
2.7\end{array}$ \\
\hline $\begin{array}{l}\text { Place of stillbirth takes place }(n=52) \\
\text { Hospital } \\
\text { Health center } \\
\text { Health post/clinic } \\
\text { Home }\end{array}$ & $\begin{array}{l}24 \\
20 \\
5 \\
3\end{array}$ & $\begin{array}{l}46.2 \\
38.5 \\
9.6 \\
5.8 \\
\end{array}$ & $\begin{array}{l}39.7 \\
37.4 \\
13.0 \\
9.9\end{array}$ \\
\hline $\begin{array}{l}\text { Attendant of stillbirth }(n=52) \\
\text { Midwifery/Nurse/HO } \\
\text { Medical Doctor } \\
\text { HEW } \\
\text { Family member }\end{array}$ & $\begin{array}{l}38 \\
6 \\
5 \\
3\end{array}$ & $\begin{array}{l}73.1 \\
11.5 \\
9.6 \\
5.8\end{array}$ & $\begin{array}{l}64.3 \\
12.8 \\
13.0 \\
9.9\end{array}$ \\
\hline $\begin{array}{l}\text { Possible cause of stillbirth }(n=52) \\
\text { Infections/Sepsis } \\
\text { Maternal malnutrition } \\
\text { Unnecessary medication } \\
\end{array}$ & $\begin{array}{l}38 \\
15 \\
12\end{array}$ & $\begin{array}{l}73.1 \\
28.8 \\
23.1\end{array}$ & $\begin{array}{l}80.7 \\
41.2 \\
17.8\end{array}$ \\
\hline $\begin{array}{l}\text { Any illness during pregnancy before event }(n= \\
\text { 52) } \\
\text { Fever/febrile illness } \\
\text { Malnutrition } \\
\text { Anemia } \\
\text { Epilepsy/Convulsion }\end{array}$ & $\begin{array}{l}25 \\
7 \\
5 \\
5 \\
2\end{array}$ & $\begin{array}{l}48.1 \\
13.5 \\
9.6 \\
9.6 \\
3.8\end{array}$ & $\begin{array}{l}62.0 \\
16.3 \\
13.0 \\
13.0 \\
3.4\end{array}$ \\
\hline
\end{tabular}


High blood pressure

Pregnancy related problems $(n=52)$

Severe abdominal pain

Excessive vaginal bleeding

Severe headache

Blurred vision

Foul smelling vaginal discharge

Shortness of breathing

Swelling of fingers, face and leg

\section{Prevalence of perinatal and neonatal mortality rate}

From the study subjects, a total of 2013 live births, 52 stillbirths, 43 early neonatal deaths, and 15 late neonatal deaths. As a result of cluster variation, weighted estimation was used for each parameter. Thus, weighted analysis was done to avoid estimation and under representation and it was found to be the rate of stillbirth was $27(95 \% \mathrm{Cl}: 26,28)$ per 1,000 pregnancies, early neonatal death was $23(95 \% \mathrm{Cl}: 21,24)$ per 1,000 live births, late neonatal death was $8(95 \% \mathrm{Cl}: 8,9)$ per 1,000 live birth. The weighted perinatal mortality rate was $48(95 \% \mathrm{Cl}: 46,49)$ per 1,000 pregnancies and neonatal mortality rate was $31(95 \% \mathrm{Cl}$ : 29,32 ) per 1,000 live births (Table 3).

Table 3: Status of perinatal and neonatal mortality in Benishangul Gumuz Region, Northwestern Ethiopia, March 2020 - January 2021

\begin{tabular}{|l|l|l|l|}
\hline Indicators & Frequency & $\begin{array}{c}\text { Rate/1000 (95\%CI) } \\
\text { (Un-weighted) }\end{array}$ & $\begin{array}{c}\text { Rate/1000 (95\%CI) } \\
\text { (Weighted) }\end{array}$ \\
\hline Total births & 2065 & & \\
\hline Total live births & 2013 & & $27(26-28)$ \\
\hline Still birth & 52 & $24(18-31)$ & $23(21-24)$ \\
\hline Early neonatal mortality & 43 & $21(16-24)$ & $8(8-9)$ \\
\hline Late neonatal mortality & 15 & $7(4-12)$ & $48(46-49)$ \\
\hline Perinatal mortality & 95 & $43(35-53)$ & $31(29-32)$ \\
\hline Neonatal mortality & 58 & $29(22-37)$ & \\
\hline
\end{tabular}

\section{Factors affecting Adverse birth outcome}

The determinant factors of adverse birth outcome were discovered using the mixed effect multilevel logistic regression model. Before running the full model, ICC ( $\rho)$ was calculated in the empty model for the outcome to make decision whether the data fit to a mixed effects multilevel model or not. Then, ICC 
( $\rho$ ) was calculated as a full model for the outcome to detect the variability attributed to clusters after controlling the individual level.

Likewise, the adverse birth outcomes, ICC $(\rho)$ was calculated in the empty model and it was found to be 0.32 , indicating that $32 \%$ of the variation was contributed by cluster variations. The test of the preference of log-likelihood versus logistic regression was statistically significant $(P<0.0001)$. Then, the full model was run by including both the cluster level and individual level variables and the ICC $(\rho)$ was increased to 0.53 . This again indicated that $53 \%$ of the variation was attributed to cluster level variables. The preferences of log-likelihood verses logistic regression was determined indicate that statistically significant $(p<0.0001)$. Hence, this is suggested that using multilevel analysis is crucial rather than logistic regression model (Table 4).

Table 4: Parameters of odds ratio and Test of Goodness-of-fit of the Mixed-effects Multilevel Models, Benishangul Gumuz Region, Northwest Ethiopia, 2021

\begin{tabular}{|c|c|c|c|c|c|}
\hline 'odels & $\begin{array}{c}\text { Fixed } \\
\text { intercept - } \\
\text { cons }(95 \% \mathrm{CI})\end{array}$ & $\begin{array}{l}\text { Random effect as } \\
\text { Level-2 variance var(- } \\
\text { cons }(95 \% \mathrm{CI}))\end{array}$ & $\begin{array}{l}\text { Intra-class } \\
\text { Correlation } \\
\text { Coefficient: } \\
\text { ICC }(\rho)\end{array}$ & $\begin{array}{l}\text { Log } \\
\text { likelihood } \\
\text { (LR)- } \\
\text { deviance }\end{array}$ & $\begin{array}{l}\text { Significance } \\
\text { Vs Logistic } \\
\text { (P-va }\end{array}$ \\
\hline \multicolumn{6}{|l|}{$\begin{array}{l}\text { rerse } \\
\text { h }\end{array}$} \\
\hline comes* & $\begin{array}{l}0.08(0.05, \\
0.12)\end{array}$ & $1.55(0.89,2.71)$ & $0.32=32 \%$ & -670.72 & $\mathrm{P}<0.0001$ \\
\hline $\begin{array}{l}\text { Empty } \\
\text { el }\end{array}$ & $\begin{array}{l}0.1(0.002, \\
2.92)\end{array}$ & $3.75(1.74,8.06)$ & $0.53=53 \%$ & -220.11 & $P<0.0001$ \\
\hline Full & & & & & \\
\hline
\end{tabular}

$P$ value less than 0.05 is statistically significant and the data fit for the multilevel model

* Multilevel regression model applied to measure the effect of factors on outcome

After adjusting for confounding effects, a multivariable mixed effects logistic regression model was performed. Then, the determinant factors of adverse birth outcome identified at cluster and individual level factors such that at the cluster level (level - 2) factors: house hold wealth index and at individual level (level - 1) factors: women educational status, history of abortion, time of $1^{\text {st }}$ ANC initiation, iron folic acid supplementation, initiate BF within one hours, pregnant related problems during labour and immediately after childbirth, presence of offensive amniotic fluid and immunizing newborn within postnatal period were found to have a statistical significant association with adverse birth outcomes.

The odds of having adverse birth outcomes among women resided in poor household wealth index (AOR $=3.3 ; 95 \% \mathrm{Cl}: 1.07,10.23)$ were three times higher than among women resided in rich household wealth 
index. Similarly, the odds of having adverse birth outcomes among women who attended high school $(A O R=6.12 ; 95 \% \mathrm{Cl}: 1.87,20.1)$ were six times higher than among women who had no formal education.

Women initiated a $1^{\text {st }}$ ANC visit between $4-6$ months of gestational age (AOR $\left.=0.38 ; 95 \% \mathrm{Cl}: 0.18,0.8\right)$, received iron folic acid supplementation during pregnancy ( $A O R=0.44 ; 95 \% \mathrm{Cl}: 0.14,0.98)$, initiate breast feeding within 1 hours ( $A O R=0.22 ; 95 \% \mathrm{Cl}: 0.10,0.50$ ) and immunized newborn within postnatal period $(A O R=0.33 ; 95 \% \mathrm{Cl}: 0.12,0.93)$ were lower to have adverse birth outcome than among women with their counterpart.

Women who had pregnant-related complications during pregnancy $(A O R=3.29 ; 95 \% \mathrm{Cl}: 1.68,4.46)$, labour and childbirth $(\mathrm{AOR}=6.08 ; 95 \% \mathrm{Cl}$ : $2.36,15.48)$ and immediately after childbirth $(\mathrm{AOR}=5.24$; $95 \% \mathrm{Cl}: 2.23,12.33)$ were higher to have adverse birth outcomes as compared with their counterpart. Similarly, women who had an offensive odder of amniotic fluid (AOR $=3.04 ; 95 \% \mathrm{Cl}$ : $1.37,6.75)$ were three times higher to have adverse birth outcome than among women who didn't have an offensive odder of amniotic fluid (Table 5).

Table 5: Multilevel Models analysis on factors associated with adverse birth outcome (stillbirth, neonatal death and neonatal illness) Benishangul Gumuz Region, Northwest Ethiopia 2021 


\begin{tabular}{|c|c|c|c|c|}
\hline \multirow[t]{2}{*}{ Determinant factors } & \multicolumn{2}{|c|}{ Adverse birth outcomes } & \multirow{2}{*}{$\begin{array}{c}\text { Crud OR } \\
95 \% \text { CI }\end{array}$} & \multirow{2}{*}{$\begin{array}{l}\text { Adjusted } \\
\text { OR } \\
95 \% \text { CI }\end{array}$} \\
\hline & No & Yes & & \\
\hline \multicolumn{5}{|c|}{ Level - 2 (Community level) variables } \\
\hline \multicolumn{5}{|c|}{ Place of Residents } \\
\hline $\begin{array}{l}\text { Urban } \\
\text { Rural }\end{array}$ & $613(85.5)$ & $104(14.5)$ & & 1 \\
\hline Rural & & & $\begin{array}{l}\text { 1.12(0.55, } \\
2.27)\end{array}$ & $\begin{array}{l}2.17(0.48, \\
9.85)\end{array}$ \\
\hline \multirow{3}{*}{$\begin{array}{c}\text { Time takes to reach HC } \\
<2 \text { Hours } \\
>=2 \text { Hours }\end{array}$} & & & & \\
\hline & $1,362(88.16)$ & $183(11.84)$ & 1 & 1 \\
\hline & & & $\begin{array}{l}\text { 1.98(1.15, } \\
3.42)\end{array}$ & $\begin{array}{l}0.96(0.32, \\
2.85)\end{array}$ \\
\hline \multicolumn{5}{|l|}{ Household wealth index } \\
\hline $1^{\text {st }}$ Quintile (Poor) & $566(84.60)$ & 103(15.40) & 1.63(0.97, & $3.3(1.07$, \\
\hline \multirow{2}{*}{$2^{\text {nd }}$ Quintile (Middle) } & 604(85.67) & $101(14.33)$ & 2.74) & 10.23) \\
\hline & 647(93.63) & $44(6.37)$ & 1.91(1.22, & 2.20 \\
\hline \multirow{2}{*}{$3^{\text {rd }}$ Quintile (Rich) } & & & 3.0) 1 & $\begin{array}{l}(0.98 \\
5.0)\end{array}$ \\
\hline & & & & 1 \\
\hline \multicolumn{5}{|c|}{ Leve-1 (individual level) variables } \\
\hline \multicolumn{5}{|l|}{ Age (Years) } \\
\hline \multirow[t]{2}{*}{$<20$} & 138(86.79) & 21(13.21) & & \\
\hline & $1,173(88.86)$ & $147(11.14)$ & $0.79(0.46$ & $0.50(0.04$, \\
\hline 1. -29 & 506(86.35) & 80(13.65) & 1.36) & 6.27) \\
\hline \multirow[t]{2}{*}{$>=30$} & & & $0.89(0.5$ & 0.35 \\
\hline & & & & $\begin{array}{l}(0.03 \\
4.67)\end{array}$ \\
\hline \multicolumn{5}{|l|}{ Ethnicity } \\
\hline \multirow{3}{*}{$\begin{array}{l}\text { Berta } \\
\text { Others }\end{array}$} & 989(90.32) & 106(9.68) & & \\
\hline & 828(85.36) & $142(14.64)$ & $0.98(0.58$ & $0.96(0.3$ \\
\hline & & & 1.66) & \\
\hline \multicolumn{5}{|l|}{ Woman education level } \\
\hline \multirow{7}{*}{$\begin{array}{l}\text { No formal education } \\
\text { Primary School } \\
\text { High School } \\
\text { Tertiary education }\end{array}$} & $1,146(89.25)$ & $138(10.75)$ & 1 & \\
\hline & $333(86.05)$ & $54(13.95)$ & 1.41(0.95, & $1.41(0.5$ \\
\hline & $180(83.33)$ & 36(16.67) & 2.1) & 3.97) \\
\hline & 158(88.76) & $20(11.24)$ & $1.52(0.96$ & 6.12(1.87, \\
\hline & & & $\begin{array}{l}2.43) \\
0.79(0.45\end{array}$ & 20.1) \\
\hline & & & $137)$ & $(022$ \\
\hline & & & & $3.77)$ \\
\hline \multicolumn{5}{|l|}{ Partner education level } \\
\hline \multirow{6}{*}{$\begin{array}{l}\text { Normal education } \\
\text { Primary School } \\
\text { High School } \\
\text { Tertiary education }\end{array}$} & $1,033(89.21)$ & $125(10.79)$ & 1 & 1 \\
\hline & 199(82.23) & $43(17.77)$ & 2.08(1.32, & 1.49(0.53, \\
\hline & 245(88.45) & $32(11.55)$ & 3.29) & \\
\hline & 286(86.93) & 43(13.07) & $0.99(0.62$ & 1.44(0.52, \\
\hline & & & 1.61) & 4.07) \\
\hline & & & $\begin{array}{l}0.84(0.54, \\
129)\end{array}$ & $0.69(0.22$, \\
\hline & & & & \\
\hline & $1.263(90.15)$ & $138(9.85)$ & 1 & 1 \\
\hline $\begin{array}{l}\text { No } \\
\text { Yes }\end{array}$ & $130(76.02)$ & $41(23.98)$ & 3.46(2.18, & 4.2(1.78, \\
\hline Information on MHS & & & & \\
\hline
\end{tabular}




\begin{tabular}{|c|c|c|c|c|}
\hline $\begin{array}{l}\text { No } \\
\text { Yes }\end{array}$ & $\begin{array}{l}150(81.97) \\
1,667(88.58)\end{array}$ & $\begin{array}{l}33(18.03) \\
215(11.42)\end{array}$ & $\begin{array}{l}1 \\
0.62(0.38, \\
0.99)\end{array}$ & $\begin{array}{c}1 \\
0.93(0.3 \\
2.89) \\
\end{array}$ \\
\hline $\begin{array}{l}\text { Place of delivery for previous } \\
\text { delivery } \\
\text { Home } \\
\text { Health Post } \\
\text { Health Center } \\
\text { Hospital }\end{array}$ & $\begin{array}{l}354(86.76) \\
322(91.48) \\
531(86.91) \\
186(92.54)\end{array}$ & $\begin{array}{l}54(13.24) \\
30(8.52) \\
80(13.09) \\
15(7.46)\end{array}$ & $\begin{array}{l}\quad 1 \\
0.82(0.44 \\
1.51) \\
0.59(0.37 \\
0.97) \\
0.33(0.16 \\
0.71)\end{array}$ & $\begin{array}{l}1 \\
1.19(0.46, \\
3.09) \\
1.26(0.55 \\
2.89) \\
0.39(0.1 \\
1.55)\end{array}$ \\
\hline $\begin{array}{l}\text { Availability of MHS } \\
\text { No } \\
\text { Yes }\end{array}$ & $\begin{array}{l}103(83.74) \\
1,714(88.26)\end{array}$ & $\begin{array}{l}20(16.26) \\
228(11.74)\end{array}$ & $\begin{array}{l}1 \\
0.75(0.42 \\
1.34)\end{array}$ & \begin{tabular}{l}
1 \\
\multicolumn{1}{c}{1} \\
$2.76(0.18$ \\
$42.85)$
\end{tabular} \\
\hline $\begin{array}{l}\text { Provision of maternal health care } \\
\text { for community } \\
\text { No } \\
\text { Yes }\end{array}$ & $\begin{array}{l}115(80.99) \\
1,702(88.51)\end{array}$ & $\begin{array}{l}27(19.01) \\
221(11.49)\end{array}$ & $\begin{array}{l}1 \\
0.59(0.36 \\
1.0)\end{array}$ & $\begin{array}{l}1 \\
1.1(0.1 \\
11.37) \\
\end{array}$ \\
\hline $\begin{array}{l}\text { Time of } 1^{\text {st }} \text { ANC initiation } \\
1-3 \text { months of GA } \\
4-6 \text { months of GA } \\
\text { After } 6 \text { months of GA }\end{array}$ & $\begin{array}{l}459(84.84) \\
1,223(92.37) \\
81(61.83)\end{array}$ & $\begin{array}{l}82(15.16) \\
101(7.63) \\
50(38.17)\end{array}$ & $\begin{array}{l}\quad 1 \\
0.31(0.21 \\
0.46) \\
4.16(2.41 \\
7.19)\end{array}$ & $\begin{array}{l}\quad 1 \\
0.38 \\
(0.18 \\
0.8) \\
0.5(0.11 \\
2.32)\end{array}$ \\
\hline $\begin{array}{c}\text { Number of ANC Visits } \\
<4 \\
>=4\end{array}$ & $\begin{array}{l}523(85.18) \\
1,294(89.18)\end{array}$ & $\begin{array}{l}91(14.82) \\
157(10.82)\end{array}$ & $\begin{array}{l}1 \\
0.69(0.49 \\
0.97)\end{array}$ & $\begin{array}{l}1 \\
0.98(0.42 \\
2.3)\end{array}$ \\
\hline $\begin{array}{c}\text { IFA supplementation } \\
\text { No } \\
\text { Yes }\end{array}$ & $\begin{array}{l}306(78.06) \\
1,511(90.32)\end{array}$ & $\begin{array}{l}86(21.94) \\
162(9.68)\end{array}$ & $\begin{array}{l}1 \\
0.31(0.22, \\
0.44)\end{array}$ & $\begin{array}{l}1 \\
1 \\
0.44(0.14, \\
0.98)\end{array}$ \\
\hline $\begin{array}{c}\text { TT during pregnancy } \\
\text { No } \\
\text { Yes }\end{array}$ & $\begin{array}{l}410(81.03) \\
1,407(90.25)\end{array}$ & $\begin{array}{l}96(18.97) \\
152(9.75)\end{array}$ & $\begin{array}{l}1 \\
0.41(0.29 \\
0.57)\end{array}$ & $\begin{array}{l}1 \\
1.08(0.41 \\
2.81)\end{array}$ \\
\hline $\begin{array}{c}\text { Initiate BF within 1hrs } \\
\text { No } \\
\text { Yes }\end{array}$ & $\begin{array}{l}303(71.97) \\
1,514(92.09)\end{array}$ & $\begin{array}{l}118(28.03) \\
130(7.91)\end{array}$ & \begin{tabular}{l}
\multicolumn{1}{c}{1} \\
$0.15(0.11$ \\
$0.22)$
\end{tabular} & $\begin{array}{l}1 \\
0.22(0.10, \\
0.50)\end{array}$ \\
\hline $\begin{array}{l}\text { Pregnant related problems during } \\
\text { pregnancy } \\
\text { No } \\
\text { Yes }\end{array}$ & $\begin{array}{l}1,525(91.37) \\
292(73.74)\end{array}$ & $\begin{array}{l}144(8.63) \\
104(26.26)\end{array}$ & \begin{tabular}{l}
1 \\
\multicolumn{1}{c}{1} \\
$3.99(2.86$, \\
$5.60)$
\end{tabular} & \begin{tabular}{l}
1 \\
\multicolumn{1}{c}{1} \\
$3.29(1.68$, \\
$6.46)$
\end{tabular} \\
\hline $\begin{array}{ll}\text { Pregnant } & \text { related problems during } \\
\text { childbirth } & \\
& \text { No } \\
& \text { Yes }\end{array}$ & $\begin{array}{l}1,640(92.71) \\
176(59.66)\end{array}$ & $\begin{array}{l}129(7.29) \\
119(40.34)\end{array}$ & 1 & 1 \\
\hline
\end{tabular}




\begin{tabular}{|c|c|c|c|c|}
\hline & & & $\begin{array}{l}\text { 10.42(7.27, } \\
14.9)\end{array}$ & $\begin{array}{l}6.08(2.36, \\
15.48)\end{array}$ \\
\hline $\begin{array}{l}\text { Husband decision making on health } \\
\text { services } \\
\qquad \begin{array}{c}\text { No } \\
\text { Yes }\end{array}\end{array}$ & $\begin{array}{l}777(82.40) \\
1,040(92.69)\end{array}$ & $\begin{array}{l}166(17.60) \\
82(7.31)\end{array}$ & $\begin{array}{l}1 \\
0.42(0.29 \\
0.58)\end{array}$ & $\begin{array}{l}1 \\
0.73(0.36 \\
1.48)\end{array}$ \\
\hline $\begin{array}{l}\text { Duration of labour } \\
<12 \text { hours } \\
\text { B/n } 12-24 \text { hours } \\
>\quad 24 \text { hours }\end{array}$ & $\begin{array}{l}1,346(92.38) \\
369(80.57) \\
102(68.0)\end{array}$ & $\begin{array}{l}111(7.62) \\
89(19.43) \\
48(32.0)\end{array}$ & $\begin{array}{l}\quad 1 \\
3.62(2.52, \\
5.19) \\
6.29(3.93 \\
9.96)\end{array}$ & $\begin{array}{l}\quad 1 \\
1.21(0.56 \\
2.63) \\
0.73(0.22 \\
2.34) \\
\end{array}$ \\
\hline $\begin{array}{c}\text { Pregnant } \quad \text { related } \\
\text { immediately after delivery } \\
\text { No } \\
\text { Yes }\end{array}$ & $\begin{array}{l}1,715(91.86) \\
102(51.52)\end{array}$ & $\begin{array}{l}152(8.14) \\
96(48.48)\end{array}$ & $\begin{array}{l}1 \\
12.15(8.1 \\
18.31)\end{array}$ & \begin{tabular}{l}
\multicolumn{1}{c}{1} \\
$5.24(2.23$ \\
$12.33)$
\end{tabular} \\
\hline $\begin{array}{l}\text { Time interval for } 1^{\text {st }} \text { PNC } \\
\text { Within 2days } \\
\text { B/n } 3-7 \text { days } \\
\text { B/n } 8 \text { - 42days }\end{array}$ & $\begin{array}{l}431(85.52) \\
682(90.45) \\
431(89.23)\end{array}$ & $\begin{array}{l}73(14.48) \\
72(9.55) \\
52(10.77)\end{array}$ & $\begin{array}{l}\quad 1 \\
0.93(0.59 \\
1.45) \\
0.91(0.5 \\
1.64)\end{array}$ & $\begin{array}{l}\quad 1 \\
0.68(0.3 \\
1.53) \\
0.75(0.27 \\
2.04)\end{array}$ \\
\hline $\begin{array}{l}\text { Time of PMRM before labour } \\
<=1 \text { hour } \\
1-12 \text { hours } \\
>12 \text { hours }\end{array}$ & $\begin{array}{l}717(88.08) \\
1,002(88.52) \\
73(77.66)\end{array}$ & $\begin{array}{l}97(11.92) \\
130(11.48) \\
21(22.34)\end{array}$ & $\begin{array}{l}\quad 1 \\
1.45(1.03 \\
2.03) \\
5.37(2.8 \\
10.27)\end{array}$ & $\begin{array}{l}1 \\
0.76(0.39 \\
1.47) \\
\quad-\end{array}$ \\
\hline $\begin{array}{c}\text { Bad odder of Amniotic fluid } \\
\text { No } \\
\text { Yes }\end{array}$ & $\begin{array}{l}1,567(89.14) \\
249(81.37)\end{array}$ & $\begin{array}{l}191(10.86) \\
57(18.68)\end{array}$ & $\begin{array}{l}1 \\
2.26(1.48, \\
3.46)\end{array}$ & $\begin{array}{l}1 \\
\text { 3.04(1.37, } \\
6.75)\end{array}$ \\
\hline $\begin{array}{c}\text { Immunized the newborn } \\
\text { No } \\
\text { Yes }\end{array}$ & $\begin{array}{l}257(73.43) \\
1,560(90.96)\end{array}$ & $\begin{array}{l}93(26.57) \\
155(9.04)\end{array}$ & $\begin{array}{l}1 \\
0.23(0.16 \\
0.32)\end{array}$ & $\begin{array}{l}1 \\
0.33(0.12, \\
0.93)\end{array}$ \\
\hline
\end{tabular}

\section{Effect of Continuity of Maternal Health Services on Perinatal death}

To reduce the potential of confounding effects, we utilized propensity score matching (PSM) approach to compare women who had complete continuum of care in maternal health services with their main interventions to those who had terminated/discontinued the services. Among the three distinct techniques of propensity score matching (PSM), one to one matching was utilized to quantify the effect of intervention on perinatal death. After matching treated and control individual, the effects of completion 
of continuum of care in maternal health services via time and space dimension on perinatal death were determined.

Hence, the result found to be received $1^{\text {st }}$ ANC visit ( $\left.\beta=-0.08 ; 95 \% \mathrm{Cl}:-0.13,-0.04 ; p<0.0001\right)$; completed $4^{\text {th }}$ ANC visit $(\beta=-0.04 ; 95 \% \mathrm{Cl}:-0.06,-0.02 ; p=0.002)$; skilled attendant of ANC services $(\beta=-0.03 ; 95 \% \mathrm{Cl}$ : $-0.05,-0.01 ; p=0.002)$; completed continuity of care for both $4^{\text {th }}$ ANC and skilled delivery $(\beta=-0.02$; $95 \% \mathrm{Cl}:-0.04,-0.01 ; p=0.006)$; completion of continuum of care in maternal health services $(\beta=-0.03$; $95 \% \mathrm{Cl}:-0.04,-0.01 ; p=0.007)$; completed key services of ANC package $(\beta=-0.04 ; 95 \% \mathrm{Cl}:-0.05,-0.02 ; p<$ $0.0001)$, completion of key services of PNC package ( $\beta=-0.05 ; 95 \% \mathrm{Cl}:-0.07,-0.04 ; p<0.0001)$, complete whole key service maternal health services $(\beta=-0.04 ; 95 \% \mathrm{Cl}:-0.05,-0.02 ; p<0.0001)$ and completed continuum of care via space dimension $(\beta=-0.02 ; 95 \% \mathrm{Cl}:-0.04,-0.002 ; \mathrm{p}=0.027)$ were associated with a significant reduction in the likelihood of perinatal death (Table 6).

Table 6: Propensity Score Matching Analysis on the effect of continuum of care in maternal health services on perinatal death Benishangul Gumuz Region, Northwest Ethiopia 2021 
Factors

\begin{tabular}{|c|c|}
\hline \multicolumn{2}{|c|}{ Perinatal death } \\
\hline No & Yes \\
\hline
\end{tabular}

Adjusted

estimate

B 95\%CI

value

I. Continuity of care in maternal health services via time dimension

First ANC services

No Received

Received

Fourth ANC services

Discontinued

Completed care

Delivery Care Services

Unskilled delivery services

Skilled delivery services

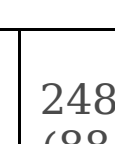

(88.89)

1,855

(96.66)

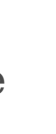




\begin{tabular}{|l|l|c|c|}
802 & 16 & $-0.02(-0.04$, & $P=$ \\
$(98.04)$ & $(1.96)$ & $-0.002)$ & 0.027 \\
\hline
\end{tabular}

\footnotetext{
* adjusted for place of resident, educational status, occupational status, household wealth index and distance of health facility
}

\section{Discussion}

\section{Status of Adverse Birth Outcome}

Adverse birth outcomes are the most essential vital data used to evaluate maternal and child health programs and plan evidence-based interventions. They serve as a gauge for the quality and effectiveness of maternal health services that provided to mothers and children. The basic indicators used to measure adverse birth outcomes were stillbirth, neonatal death and any illness during postnatal period. Thus, this study found that $12.4 \%$ women had adverse birth outcomes which was lower than evidence from Northern Ethiopia $27.5 \%(20)$. This is because the variation of study time and design. Due to the time variation, a community based intervention that implemented by HEW will reduce the magnitude of adverse birth outcomes in the study area.

According to the global target, every country will have 12 or fewer stillbirths per 1,000 births by 2030 . Even though developed countries and upper middle income countries were meet their target, developing and low income countries will have to more than double present progress to reach this target (21). Beside these, magnitude of stillbirth was 27 stillbirths per 1,000 pregnancies in the study area. It was much higher than global target (12 per 1,000 pregnancies), Mumbai Slums 8.68 per 1,000 births (22) and Ghana 1.5\% (23). But, it was lower than study done in Rural Pakistan 39.2 per 1,000 birth (24), Northern Ethiopia 8.1 \% (20), Southwest Nigeria 4.7\% (25), Gondar University Hospital 7.1\% (26). However, this finding was consistent with evidence from rural south western Uganda $2.4 \%(27)$, Rural Uganda $2.4 \%(27)$ and Tigrary region (2.3\%) (28). The reason may be reduction of stillbirth is much slower than other countries due to poor utilization of recommended ANC services via visit based and content based continuity of care and also the quality of maternal health services were poor as compared with other countries.

Neonatal mortality is the probability of dying within the first month of life which is the most pertinent national indicator in maternal and child health programs. Hence, this study found that neonatal mortality rate was 31 per 1,000LBs. This finding is almost similar with the evidence from EDHS 2019 (33 per 1,000LBs)(29), Ghana 30.85 per 1,000LBs(30) and Indian State of Bihar 32.2 per 1,000LBs (31). However, it was lower than evidence from Rural Pakistan 49.65 per 1,000LBs (24), Tigerary region 62.5 per 1000LBs)(32) and Jimma Zone 35.5 per 1,000LBs(18). But, it was much higher than study in Mumbai Slums 13.98 per live births (22), Vietnam 11.65 per 1,000LBs(33), India 12 deaths per 1,000LBs(7) and Tigrary $4.6 \%(28)$. This implies that neonatal mortality is still high in developing countries and Ethiopia particularly in the study area and there is no any improvement in the reduction of neonatal mortality to achieve global and national targeted goal. 
In this study, we found that perinatal mortality rate was 48 per 1,000 pregnancies. This finding is consistent with the pooled estimate of perinatal mortality rate in Ethiopia was 51.3 per total births(34). However, this finding was higher than study in Hawassa town HF 2.7\% (35) and rural northern Ghana 39 per 1,000 births(36). Whereas, it was lower than evidence from low income countries 55 per 1,000 births (37), Rural Pakistan 69.95 per birth (24) and Rural Congo 61 per 1,000 births(38). Among newborns died within postnatal periods, $59.5 \%$ of the newborns were died within 48 hours and early neonatal deaths contribute $72.9 \%$ of neonatal deaths. This finding is consistent with different study done in the countries and outside the countries $(32,33,37,39)$.

Worldwide, the major causes of neonatal death are infections (sepsis/pneumonia, tetanus and diarrhea), prematurity/preterm birth and birth asphyxia. In line with these, we found that the three major neonatal characteristics that causes neonatal death: birth asphyxia (66.0\%), sepsis/infection (50.5\%) and pneumonia (42.0\%). This finding was supported by different studies $(18,22,36-40)$. However, some variation between countries may be sought because of the variability of quality, availability and utilization rate of maternal and newborn health services.

Maternal complications during pregnancy and childbirth are predisposing factors for neonatal death. As result, safe motherhood is a proven initiatives to prevent newborn death by overcoming pregnant related complications(39). Hence, this study found that obstructed labour (56.4\%), obstetric sepsis/maternal infection (54.7\%), and APH (33.3\%) were common pregnant related problems that cause neonatal death. This finding was consistent with evidence from low and middle income countries which found that obstructed labor and placental abruption cause birth asphyxia, and also maternal infections cause neonatal infections that cause neonatal death (41). This is due maternal and neonatal health condition are interlinked each other's.

In this study, the possible causes of stillbirth were maternal infection or obstetric sepsis, maternal malnutrition and unnecessary medication. This finding is consistent with evidence from low-income countries (37) and rural northwest Bangladesh (42). Moreover, before the occurrence of stillbirth, women were frequently suffered with pregnant related problems (severe abdominal pain, excessive vaginal bleeding and blurred vision and any illness during pregnancy (febrile illness, malnutrition and anemia). This finding is supported by other study (21). This is because pregnant related problem and maternal illness during pregnancy cause poor placental function, either with fetal growth restriction or preterm labor, or both, is a common cause of stillbirths. As a result, different evidence argued that better prenatal care is critical to preventing 1.3 million stillbirths and ending unnecessary neonatal deaths (21).

\section{Determinant factors of Adverse Birth Outcome}

Among community level factors, household wealth index is predictor of adverse birth outcome. Beside, women resided in poor household wealth index were three times more likely to have adverse birth outcome. This is because level of household index are directly related to access of information and receiving health services, which enable them to prevent themselves and her newborn from any illness and adverse event. 
Educational status is a determinant factor of adverse birth outcomes, in which women who attended high school were six times more likely to had adverse birth outcome as compared with women who had no formal education. This may be educated people have better knowledge and information on the impact of adverse birth outcomes, as result they are early diagnosis the problems and report properly for further treatment and management as compared with people with no formal education. Moreover, people lack education did not report any adverse event and believing that the event as a usual life rather than considering as health problems. So, the magnitude of adverse birth outcomes was high among educated people.

In this study, initiating 1st ANC visit between $4-6$ months of gestational age reduced adverse birth outcomes by $62 \%$. This study is consistent with previous study in Gondar University Hospital (26) and Ghana (23). This could be the fact that during ANC visits, relevant health issues can be identified and addressed early. This is only feasible if pregnant women present themselves to a suitable health facility at an early stage of labor and pregnancy. As a result, the probability of occurring adverse birth outcomes will be remission.

According to WHO directives, IFA supplementation during pregnancy, early initiating BF within 1 hour for the newborn and immunization increased the survival of newborn by overcoming the adverse event. In line these, we found that women received IFA supplementation during pregnancy and initiate BF within 1hours for her newborn and immunized newborn within postnatal period were less likely to have adverse birth outcomes. This finding is concurrent with previous studies within the country and abroad $(6,10,18$, 32).

The occurrence of pregnant related problems during pregnancy, childbirth and immediately after childbirth decreases the survival of newborn. Beside, we depicted that women who had pregnant-related complications during pregnancy, labour and childbirth and immediately after childbirth were more likely to have adverse birth outcomes. This finding consistent with evidence from low- and middle-income countries(43), Developing countries(44), Ghana(23), Indian State of Bihar(31), Jimma Zone(18) and Gondar University Hospital(26). This is the fact that women who have pregnant related complications were prone for infection and birth asphyxia which are the leading cause of stillbirth, neonatal death and neonatal illness. This is also explained by the health condition of newborn relies on maternal health status before, during and after conception.

As principle, amniotic fluid should be odorless or have a slightly sweet odor. However, if it is noticed that smells bad, it could be a sign and symptom of uterine infection. Thus, this study revealed that women who had an offensive odder of amniotic fluid were three times more likely to have adverse birth outcome. This is explained by offensive odder of amniotic fluid is an indication of infections, which is the leading cause of stillbirth birth, neonatal death and illness during postnatal period.

\section{Effect of continuum of care in maternal health services on perinatal death}


Maternal health interventions during pregnancy, childbirth and after childbirth have a beneficial health impact on maternal and newborn health outcomes (45). This is because early detection of pregnancyrelated disorders throughout pregnancy, management of labor and delivery, care of any adverse event, and treatment of serious infectious infections and acute malnutrition are all effective interventions of maternal health services packages, which could reduce $77 \%$ of perinatal mortality $(46)$.

In line with these, we found that women received 1st ANC visit; completed 4th ANC visit; skilled attendant of ANC services; completed continuity of care for both 4th ANC and skilled delivery; completion of continuum of care in maternal health services; completed key services of ANC package, completion of key services of PNC package, complete whole key service maternal health service and completed continuum of care via space dimension were significantly reduce perinatal deaths. This finding is consistent with previous studies conducted in the country and abroad $(6,7,18,23,24,28,38,40,47-60)$. This is because more than one third of all child deaths occur in the first month of life, providing skilled care to mothers during pregnancy, as well as during and after birth, greatly contributes to child survival, with two-thirds of newborn deaths preventable if known, effective health measures are provided at birth and during the first week of life.

\section{Conclusions}

In this study, we concluded that neonatal and perinatal mortality rate were high as compared with national and international targets. Risk factors for adverse birth outcomes were poor household wealth index, pregnant related complication during pregnancy, childbirth and immediately after childbirth, bad odder amniotic fluid and history of stillbirth. Whereas, the protective interventions were receiving IFA supplementation during pregnancy, initiating BF within 1 hour for newborn, immunizing newborn and completion of continuity of care in maternal health services via time and space dimension. Efforts should be made to strengthening supplementation of IFA during, immunizing newborn and early initiation of BF for newborn as well as early detection and treatment of risk factors for poor birth outcomes were strongly recommended. Moreover, increased attention to newborns, as well as, in the context of the continuum of care, to women before and after giving birth, is required to effectively reduce adverse birth outcomes, perinatal and neonatal mortality. This must be accomplished through reaching out to disadvantaged populations' particularly poor population and providing them with the necessary health care.

\section{Abbreviations}

ANC

Antenatal Care

AOR

Adjusted Odd Ratio

APH

Antepartum Hemorrhage

BF 
Breast Feeding

BGRS

Benishangul Gumuz Region

Cl

Confidence Interval

COC

Continuum of Care

COR

Crude Odd Ratio

EDHS

Ethiopia Demographic and Health Survey

FSU

Final Sampling Unit

GA

Gestational Age

HC

Health Center

HEW

Health Extension Workers

$\mathrm{HO}$

Health Officer

ICC

Intra-class Correlation Coefficient

IFA

Iron Folic Acid

IRB

Institutional Review Board

LBs

Live Births

LR

Log Likelihood Ratio

$\mathrm{MCH}$

Maternal and Child Health

MHS

Maternal Health Services

MPDSR

Maternal and Perinatal Death Surveillance and Response

NMR

Neonatal Mortality Rate

PCA 
Principal Component Analysis

PNC

Postnatal Care

PSM

Propensity Score Matching

REC

Research Review and Ethics Committee

SPH

School of Public Heath

SPSS

Statistical Package for Social Sciences

SRS

Simple Random Sampling

TT

Tetanus Toxoid

WHO

World Health Organization

\section{Declarations}

\section{Ethics approval and consent to participate}

Ethical approval was obtained from Research Review and Ethics Committee (REC) of School of Public Health, Addis Ababa University's with protocol number SPH/3089/011 and Institutional Review Board (IRB) of College of Health Sciences of Addis Ababa University with protocol number 048/19/SPH. Necessary permission letters were obtained from Regional Health Bureau and respective all local districts. Confidentiality was maintained by avoiding any identities from the questionnaire. Before starting actual data collection, written and verbal consent was obtained from each study subjects.

\section{Consent to publish}

Not Applicable

\section{Availability of data and materials}

The datasets used and analyzed during the current study are available from the corresponding author on reasonable request.

\section{Competing interests}


The authors declare that they have no competing interests.

\section{Funding}

This study funded by Addis Ababa University but there is no other funding agencies for this works

\section{Authors' contributions}

MA conceived and designed the study. Then after, data was collected, analyzed, interpreted and wrote the whole document. $A W$ and $G T$ were critically commenting the whole document and genuinely guide the whole work. All authors read and approved the final manuscripts.

\section{Acknowledgements}

We would like to thank Addis Ababa University, College of Health for providing ethical approval and partially sponsored this project work. The authors would also want to express their gratitude to all of the pregnant women, data collectors, supervisors, and other individuals who contributed directly or indirectly to the study's success. Last but not least, the author wishes to express his gratitude to Miss Abebech Tefera, Miss Chaltu Argeta, Dr. Nigatu Disassa, and Assistant professor Atnafu Morka for their unwavering assistance in the preparation, magement and duplication of research questionnaires.

\section{References}

1. Marian F. MacDorman TJM, Ashna D. Mohangoo. International Comparisons of Infant Mortality andRelated Factors: United States and Europe. September, 2014;63(5).

2. Website:. https://www.idrc.ca/en/initiative/innovating-maternal-and-child-health-africa.

3. Trend in Maternal Mortality:1990 to 2015 Estimates2015; Geneva, Switzerland: World Health Organization (WHO).

4. De Graft-Johnson J KK, Tinker A, et al. The maternal, newborn and child health continuum of care: Opportunities for Africa's newborns. Partnership for Maternal, Newborn and Child Health. 2011;2006:23-36.

5. Joseph de Graft-Johnson KK, Anne Tinker, Susan Otchere, Indira Narayanan, Rumishael Shoo,Doyin Oluwole, Joy Lawn. Household to home continuum of care for maternal, newborn, and child health.24 - 33.

6. Kayode GA, Ansah E, Agyepong IA, et al. Individual and community determinants of neonatal mortality in Ghana: a multilevel analysis. BMC Pregnancy and Childbirth. 2014;14:165-.

7. Fadel SA, Ram U, Morris SK, et al. Facility Delivery, Postnatal Care and Neonatal Deaths in India: Nationally-Representative Case-Control Studies. PLoS One. 2015;10(10):e0140448. 
8. Carvajal-Aguirre L, Amouzou A, Mehra V, et al. Gap between contact and content in maternal and newborn care: An analysis of data from 20 countries in sub-Saharan Africa. J Glob Health. 2017;7(2):020501.

9. Manandhar DS, Osrin D, Shrestha BP, et al. Effect of a participatory intervention with women's groups on birth outcomes in Nepal: cluster-randomised controlled trial. The Lancet. 2004;364(9438):970-9.

10. Kumar V, Mohanty S, Kumar A, et al. Effect of community-based behaviour change management on neonatal mortality in Shivgarh, Uttar Pradesh, India: a cluster-randomised controlled trial. The Lancet. 2008;372(9644):1151-62.

11. Kikuchi K AE, Okawa S, Enuameh Y, Yasuoka J, Nanishi K, et al. Effective Linkages of Continuum of Care for Improving Neonatal, Perinatal, and Maternal Mortality: A Systematic Review and MetaAnalysis. PLoS ONE. 2015;10(9).

12. Azad K, Barnett $S$, Banerjee $B$, et al. Effect of scaling up women's groups on birth outcomes in three rural districts in Bangladesh: a cluster-randomised controlled trial. The Lancet. 2010;375(9721):1193-202.

13. ((UNDP) UNDP-E. The Benishangul Regional State is selected for design of model REDD program under the UNFCCC Warsaw Framework. 2017.

14. National Institution for Transforming India (NITI) Aayog, Goverment of India, 2013. Website: http://niti.gov.in/content/neo-natal-mortality-rate-nmr-1000-live-births [Internet].

15. Agency CS, editor Ethiopia Demographic and Health Survey2016; Addis Ababa: The DHS Program ICF Rockville, Maryland, USA.

16. $(\mathrm{MoH}) \mathrm{EMoH}$. National Technical Guidance For Mataernal and Perinatal Death Surveillance and Response (MPDSR). In: INSTITUE EPH, editor. 2017.

17. Agha S WE. Maternal and Child Health Program Indicator Survey 2013, Sindh Province. MNCH Services Component, USAID/Pakistan MCH Program. Karachi, Pakistan: Jhpiego. 2013.

18. Gurmesa Tura Debelew, Mesganaw Fantahun Afework, Yalew AW. Determinants and Causes of Neonatal Mortality in Jimma Zone, Southwest Ethiopia: A Multilevel Analysis of Prospective Follow Up Study. PLoS ONE. 2014;9(9).

19. Yaliso Yaya Balla TD, Bernt Lindtjorn. Maternal and neonatal mortality in rural south Ethiopia:Outcomes of Community-Based Birth Registration by Health Extension Workers. January, 2015;10(3).

20. Seyoum E, Bekele A, Tsegaye AT, et al. Magnitude and Determinants of Adverse Perinatal Outcomes in Tefera Hailu Memorial Hospital, Sekota Town, Northern Ethiopia. Global pediatric health. 2021;8:2333794x211015524.

21. Joy E Lawn, Hannah Blencowe, Peter Waiswa, et al. Ending preventable stillbirths: Stillbirths: rates, risk factors, and acceleration towards 2030. The Lancet Ending Preventable Stillbirths Series with The Lancet Stillbirth Epidemiology.Lancet 2016; 387: 587-603.

22. Gülmezoglu AM, More NS, Bapat U, et al. Community Mobilization in Mumbai Slums to Improve Perinatal Care and Outcomes: A Cluster Randomized Controlled Trial. PLoS Medicine. 
2012;9(7):e1001257.

23. Afulani PA. Determinants of stillbirths in Ghana: does quality of antenatal care matter? BMC Pregnancy Childbirth. 2016;16(1):132.

24. Bhutta ZA, Soofi S, Cousens $S$, et al. Improvement of perinatal and newborn care in rural Pakistan through community-based strategies: a cluster-randomised effectiveness trial. The Lancet. 2011;377(9763):403-12.

25. Awoleke JO, Olofinbiyi BA. Poor prenatal service utilization and pregnancy outcome in a tertiary health facility in Southwest Nigeria. Pan Afr Med J. 2020;35:28-.

26. Adane AA, Ayele TA, Ararsa LG, et al. Adverse birth outcomes among deliveries at Gondar University Hospital, Northwest Ethiopia. BMC Pregnancy Childbirth. 2014;14:90.

27. Asiki; G, Baisley; K, Newton; R, et al. Adverse pregnancy outcomes in rural Uganda (1996-2013): trends and associated factors from serial cross sectional surveys. BMC Pregnancy and Childbirth. 2015;15:279.

28. Haftu A, Hagos H, Mehari MA, et al. Pregnant women adherence level to antenatal care visit and its effect on perinatal outcome among mothers in Tigray Public Health institutions, 2017: cohort study. BMC Res Notes. 2018;11(1):872.

29. Ethiopian Public Health Institute E, Federal Ministry of Health F, Icf. Ethiopia Mini Demographic and Health Survey 2019. Addis Ababa, Ethiopia: EPHI/FMoH/ICF, 2021.

30. Betty R Kirkwood, Alexander Manu, Augustinus H A ten Asbroek, et al. Eff ect of the Newhints homevisits intervention on neonatal mortality rate and care practices in Ghana: a cluster randomised controlled trial. Lancet 2013; 381 2184-92: Website: http://dxdoiorg/101016/S0140-6736(13)600951. 2013.

31. Kumar GA, Dandona R, Chaman P, et al. A population-based study of neonatal mortality and maternal care utilization in the Indian state of Bihar. BMC Pregnancy and Childbirth. 2014;14:357.

32. Mengesha HG, Wuneh AD, Lerebo WT, et al. Survival of neonates and predictors of their mortality in Tigray region, Northern Ethiopia: prospective cohort study. BMC Pregnancy and Childbirth. 2016;16:202.

33. Eriksson L, Nga NT, Hoa DTP, et al. Secular trend, seasonality and effects of a community-based intervention on neonatal mortality: follow-up of a cluster-randomised trial in Quang Ninh province, Vietnam. J Epidemiol Community Health. 2018;72(9):776-82.

34. Jena BH, Biks GA, Gelaye KA, et al. Magnitude and trend of perinatal mortality and its relationship with inter-pregnancy interval in Ethiopia: a systematic review and meta-analysis. BMC pregnancy and childbirth. 2020;20(1):432-.

35. Tsegaye B, Kassa A. Prevalence of adverse birth outcome and associated factors among women who delivered in Hawassa town governmental health institutions, south Ethiopia, in 2017. Reprod Health. 2018;15(1):193.

36. Engmann C, Walega P, Aborigo RA, et al. Stillbirths and early neonatal mortality in rural Northern Ghana. Tropical Medicine \& International Health. 2012;17(3):272-82. 
37. Engmann C, Garces A, Jehan I, et al. Causes of community stillbirths and early neonatal deaths in low-income countries using verbal autopsy: an International, Multicenter Study. J Perinatol. 2012;32(8):585-92.

38. Matendo RM EC, Ditekemena JD, Gado J, Tshefu A, McClure EM et al. Challenge of Reducing Perinatal Mortality in Rural Congo: Findings of a Prospective, Population-based Study. Journal of Health, Population and Nutrition. 2011;29(5):532-40.

39. Joy E Lawn, Simon Cousens, Jelka Zupan. Neonatal Survival and neonatal deaths: When? Where? Why? The Lancet Neonatal Survival Steering Team. 2005;Lancet 2005; 365: 891-900.

40. Fottrell E, Azad K, Kuddus A, et al. The effect of increased coverage of participatory women's groups on neonatal mortality in Bangladesh: A cluster randomized trial. JAMA Pediatr. 2013;167(9):816-25.

41. Goldenberg RL, McClure EM, Saleem S. Improving pregnancy outcomes in low- and middle-income countries. Reprod Health. 2018;15(Suppl 1):88.

42. Sikder SS, Labrique AB, Craig IM, et al. Patterns and determinants of care seeking for obstetric complications in rural northwest Bangladesh: analysis from a prospective cohort study. BMC Health Serv Res. 2015;15:166.

43. Harrison MS, Ali S, Pasha O, et al. A prospective population-based study of maternal, fetal, and neonatal outcomes in the setting of prolonged labor, obstructed labor and failure to progress in lowand middle-income countries. Reprod Health. 2015;12 Suppl 2:S9.

44. Firdawek E, Worku A. Maternal Near Miss and Still birth in developing countries: A Systematic Review with Meta-Analysis. Journal of Pregnancy and Child Health. 2015;02(06).

45. Lassi ZS, Majeed A, Rashid S, et al. The interconnections between maternal and newborn healthevidence and implications for policy. J Matern Fetal Neonatal Med. 2013;26 Suppl 1:3-53.

46. Black RE, Levin C, Walker N, et al. Reproductive, maternal, newborn, and child health: key messages from Disease Control Priorities 3rd Edition. The Lancet. 2016;388(10061):2811-24.

47. Gurmesa Tura, Mesganaw Fantahun, Worku A. The effect of health facility delivery on neonatal mortality: systematic review and meta-analysis. BMC Pregnancy and Childbirth 2013, 13:18 Available at http://wwwbiomedcentralcom/1471-2393/13/18. 2013.

48. Aboubaker S, Qazi S, Wolfheim C, et al. Community health workers: A crucial role in newborn health care and survival. Journal of global health. 2014;4(2):020302-.

49. Abdul Hakeem Jokhio, Heather R. Winter, Kar Keung Cheng. An Intervention Involving Traditional Birth Attendants and Perinatal and Maternal Mortality in Pakistan. The new england journal of medicine: 352; 20. 2005.

50. Worku AG, Yalew AW, Afework MF. The contributions of maternity care to reducing adverse pregnancy outcomes: a cohort study in Dabat district, northwest Ethiopia. Matern Child Health J. 2014;18(6):1336-44.

51. Kikuchi K, Yasuoka J, Nanishi K, et al. Postnatal care could be the key to improving the continuum of care in maternal and child health in Ratanakiri, Cambodia2018. e0198829 p. 
52. Kananura RM, Wamala R, Ekirapa-Kiracho E, et al. A structural equation analysis on the relationship between maternal health services utilization and newborn health outcomes: a cross-sectional study in Eastern Uganda. BMC Pregnancy Childbirth. 2017;17(1):98.

53. Shiferaw K, Mengiste B, Gobena T, et al. The effect of antenatal care on perinatal outcomes in Ethiopia: A systematic review and meta-analysis. PloS one. 2021;16(1):e0245003-e.

54. Baqui AH, El-Arifeen S, Darmstadt GL, et al. Effect of community-based newborn-care intervention package implemented through two service-delivery strategies in Sylhet district, Bangladesh: a clusterrandomised controlled trial. The Lancet. 2008;371(9628):1936-44.

55. Baqui $\mathrm{AH}$, Williams EK, Rosecrans $\mathrm{AM}$, et al. Impact of an integrated nutrition and health programme on neonatal mortality in rural northern India. Bulletin of the World Health Organization. 2008;86(10):796-804.

56. Gogia S, Sachdev HP. Home-based neonatal care by community health workers for preventing mortality in neonates in low- and middle-income countries: a systematic review. J Perinatol. 2016;36 Suppl 1:S55-73.

57. Jokhio AH, Winter HR, Cheng KK. An Intervention Involving Traditional Birth Attendants and Perinatal and Maternal Mortality in Pakistan. New England Journal of Medicine. 2005;352(20):2091-9.

58. Kikuchi K, Ansah EK, Okawa S, et al. Effective Linkages of Continuum of Care for Improving Neonatal, Perinatal, and Maternal Mortality: A Systematic Review and Meta-Analysis. PLoS One. 2015;10(9):e0139288.

59. Kikuchi K, Okawa S, Zamawe CO, et al. Effectiveness of Continuum of Care-Linking Pre-Pregnancy Care and Pregnancy Care to Improve Neonatal and Perinatal Mortality: A Systematic Review and Meta-Analysis. PLoS One. 2016;11(10):e0164965.

60. Rahman A, Moran A, Pervin J, et al. Effectiveness of an integrated approach to reduce perinatal mortality: recent experiences from Matlab, Bangladesh. BMC Public Health. 2011;11:914. 